OPEN ACCESS

Edited by:

Carsten Eickhoff,

Brown University, United States

Reviewed by:

Amy Vassallo,

University of New South

Wales, Australia

Asif Ali Laghari,

Sindh Madressatul Islam

University, Pakistan

${ }^{*}$ Correspondence:

Weisi Liu

40590793@qq.com

tThese authors have contributed equally to this work and share first authorship

Specialty section:

This article was submitted to

Digital Public Health,

a section of the journa

Frontiers in Public Health

Received: 04 February 2021

Accepted: 04 June 2021

Published: 03 August 2021

Citation:

Ma X, Lu J and Liu W (2021)

Influencing Factors on Health Information to Improve Public Health

Literacy in the Official WeChat Account of Guangzhou CDC.

Front. Public Health 9:657082.

doi: 10.3389/fpubh.2021.657082

\section{Influencing Factors on Health} Information to Improve Public Health Literacy in the Official WeChat Account of Guangzhou CDC

\author{
Xiaowei $\mathrm{Ma}^{1 \dagger}$, Jianyun $\mathrm{Lu}^{2 \dagger}$ and Weisi $\mathrm{Liu}^{3 *}$ \\ ${ }^{1}$ Department of Public Health Emergency Preparedness and Response, Guangzhou Center for Disease Control and \\ Prevention, Guangzhou, China, ${ }^{2}$ Department of Infectious Disease Control and Prevention, Guangzhou Center for Disease \\ Control and Prevention, Guangzhou, China, ${ }^{3}$ Department of Health Education and Promotion, Guangzhou Center for \\ Disease Control and Prevention, Guangzhou, China
}

Background: Social media is used as a new channel for health information. In China, the official WeChat account is becoming the most popular platform for health information dissemination, which has created a good opportunity for the Centers for Disease Control and Prevention to facilitate health information online to improve emergency public health literacy.

Methods: Data were collected from the Guangzhou CDC i-Health official WeChat account between April 1, 2018 and April 30, 2019. Descriptive analysis was performed for basic information about the followers and posts of the official WeChat account. Multiple logistic regression analysis was used to analyze the association among various factors of posts on engagement of followers of the official WeChat account.

Results: Among 187,033 followers, the total numbers of post views, shares, likes, add to favorites, and comments for 213 posts were 1,147,308, 8,4671, and 5,535, respectively. Engagement of followers peaked on the dissemination date and gradually declined. The main post topics were health education posts and original posts. In the multiple logistic regression model, the number of post views was found to be significantly associated with infectious disease posts (AOR: 3.20, 95\% Cl: 1.16-8.81), original posts (AOR: 10.20, 95\% Cl: 1.17-89.28), and posts with title-reflected content (AOR: 2.93, 95\% Cl: 1.16-8.81).

Conclusion: Our findings facilitate the government to formulate better strategies and improve the effectiveness of public information dissemination.

Keywords: WeChat, official account, social media, health information, public health emergency literary

\section{INTRODUCTION}

Social media is becoming the most important channel for the public to obtain health information (1-3). Meanwhile, the government has tended to use social media to improve public health literacy to prevent infectious diseases $(4,5)$. Social media platforms such as Facebook (6-8), Twitter $(9,10)$, and Instagram $(10,11)$ were wildly used for health information. WeChat, a free mobile 
app released in 2011, is the most popular social media platform in China, and it had 1.04 billion monthly active users worldwide by the first quarter of 2018 , and $93 \%$ of residents in major Chinese cities are reported to log into WeChat daily (12). WeChat is widely used in many scenes, such as traffic classification (13), health information (12), and so on. A recent national survey in China found that one-third of participants regularly read health information articles on WeChat, and $98.53 \%$ of participants chose to use WeChat for health information seeking, indicating that the official WeChat account (OWA) is the most popular platform for health information acquisition in China (12). Due to growing online health-seeking behaviors, increasing numbers of non-authorized social media accounts are sharing biased or inaccurate health information, it is urgent for health organizations to engage with users on social media in a strategic and technological way $(14,15)$. WeChat, Twitter, and Facebook, the three most popular social media platforms globally, differ substantially in functionality, including text content, images, video, and setting of post objects $(1,10,11,16,17)$. OWA is an application account supplied by administrators on the WeChat public platform, which can be used for communication and interaction with specific groups via text, pictures, voice, and video. Members of the public can follow OWAs of interest to receive relevant information or messages. The OWA of the CDC is considered as an appropriate and professional platform for informing, educating, and empowering the public regarding health issues (18-20).

At present, a lot of studies about the influencing factors on health information by social media, including Facebook, Twitter, and Instagram, have been reported (14, 21-23). However, there are few such studies about the influencing factor of health information on an OWA (24). Studies focused on the engagement of followers and influence on the OWA of the CDC is sparse. Hence, it is important to explore the influencing factors mentioned above to get a better strategy to disseminate health information. The Guangzhou Center for Disease Control and Prevention i-Health official WeChat account (GZCDC i-Health OWA) opened in April 2018 and is managed by specialized staff at the GZCDC. Posts published via this OWA are mainly original, and focus on health tips and popular science. In our study, we analyzed the data from the posts published between April 2018 and April 2019 to explore the key factors on health information by the GZCDC i-Health OWA to improve the strategies toward health information and promote public health literacy.

\section{MATERIALS AND METHODS}

\section{Data Collection and Logging}

Data were collected from the GZCDC i-Health OWA which disseminates health information to the public. Gender and regional distribution were indicated in individual profiles. There

\footnotetext{
Abbreviations: OWA, official WeChat account; CDC, disease control and prevention; GZCDC, Guangzhou Center for Disease Control and Prevention; GZCDC i-Health OWA, Guangzhou Center for Disease Control and Prevention iHealth official WeChat account; AOR, adjusted odds ratio; 95\% CI, 95\% confident interval.
}

are three main ways for WeChat users to read the articles. First, users can get health information directly from OWAs they follow. Second, users can receive health information through "Moments," a functional mode of WeChat by which users can see their friends' posts. Finally, users can read the articles transmitted by friends. According to the operational rules, an administrator can only post once a day, with one or more posts each time. The administrator can also select a post to be the headline post. Two investigators, who received training regarding the purpose of the study and the data collection procedures, used the same standard for classification of the variables throughout the study. We used double logging of data and conducted a consistency check for the collected data.

\section{Inclusion Criteria}

All posts published by the GZCDC i-Health OWA dated from April 1, 2018 to April 30, 2019 (end of data collection) were included in this study.

\section{Variable and Characteristics}

All information was classified into different types of variables. The categorical variables included gender and regional distribution of followers, types and characteristic of posts, effectiveness of dissemination, headline, and title-reflected content. Types of posts were categorized into health education posts and organizational promotion posts. Health information posts include information or news articles on a range of health topics delivered to the public or professionals, while organizational posts cover content or messages designed to advertise or build the image of an organization or publish a notification. In other words, organizational promotion posts do not contain any health content. Based on the content of posts, we divided health information posts into six subcategories, including infectious disease, vaccine-associated event, environment health, nutrition and food-borne disease, child and adolescent health, and chronic disease, then we evaluated the most effective type of post for engaging followers. According to the copyright by the GZCDC, the posts were categorized into original posts and reposts. The position of posts was categorized into headline posts and non-headline posts. The effectiveness of posts was categorized depending on whether posts ran hot spots or not. "Running hot spots" refers to choosing topics that are close to news hot spots, addressing the health content of the audience's current concerns, and conveying the most important viewpoints and accurate information to the audience as quickly as possible (25). Whether the title reflected the content was the last independent variable. If the post content could be judged from the title, it was categorized as "Yes", and if not, it was categorized as "No."

In addition, continuous variables included the number of followers and the engagement of followers 7 days after the post was published, including the number of post views, reposts, and adding to favorites for each post. Engagement of followers was defined as the total number of interactive behaviors of followers, including views of each post, amounts of sharing, and adding to favorites. 


\section{Statistical Analysis}

Descriptive statistical analysis was performed for the continuous variables. We performed multiple logistic regression, which was utilized in previous studies $(14,21,26)$, to explore the influence factors on the engagement of followers of GZCDC i-Health OWA. We transformed a continuous dependent variable into a binary variable, because the data did not follow a normal distribution. For the engagement of followers, the median volume was used as the cut-off point to distinguish "good engagement" (equal to or larger than the median) and "poor engagement" (less than the median). For the type of posts, organizational posts that did not contain health content were set as the reference. For the characteristics of posts, reposts were set as the reference. For the effectiveness, the comment event was chosen as the reference. For the headline and whether the title reflects the content section, non-headlines and titles that did not reflect the content were set as the reference. All independent variables were included with a forced entry method. Adjusted odds ratios (ORs) and corresponding 95\% confidence intervals (CIs) for the factors were computed. $P<0.05$ were defined as statistically significant. All analyses were conducted using R software 3.4.2.

\section{Ethics Approval}

This study was approved by the ethics committee of the Guangzhou CDC. Our study did not involve any private and personal information. All data were anonymous.

\section{RESULTS}

The GZCDC i-Health OWA had 187,033 followers in April 2019, compared to 55,702 in April 2018. Among these, 24.94\% $(n=$ $46,646)$ were men, and $75.06 \%(n=140,378)$ were women. A total of $95.87 \%$ of followers $(n=182,154)$ were in Guangdong Province, and $4.13 \%$ followers $(n=4,898)$ were located in other provinces and cities. Figure 1 shows the increased cumulative followers of the Guangzhou i-Health official WeChat account from April 2018 to April 2019. The GZCDC WeChat account posted 213 posts from April 2018 to April 2019. Overall, 23.47\% of posts were organizational promotion posts, while $76.53 \%$ were health information posts, which were classified into six subcategories for further study. In addition, $91.55 \%$ of posts were original posts, $87.32 \%$ of posts were headline posts, $22.54 \%$ of posts were focused on a current event, and $46.48 \%$ of posts were reposted by other OWAs.

The total numbers of post views, shares, and adds to favorites for 213 posts were 1,147,308, 84,671, and 5,535, respectively. The proportions of direct posts, WeChat friend's circles, and friends sharing were $48.18 \%(552,804 / 1,147,308$, 95\% CI: $48.09 \%-48.14 \%), \quad 27.72 \% \quad(318,003 / 1,147,308,95 \%$ CI: $27.64 \%-27.80 \%)$, and $24.10 \%(276,501 / 1,147,308,95 \% \mathrm{CI}$ : $24.02 \%-24.18 \%$ ), respectively. The number of post views, reposts, and adds to favorites decreased simultaneously after peaking on the day of posting. Figure 2 shows the engagement of followers 7 days after posting. Table 1 reveals the characteristics about the engagement of followers to posts by the Guangzhou I-Health OWA. For post views, the highest median was chronic disease posts, and the highest maximum was infectious disease posts. Regarding the number of reposts, the highest median was child and adolescent health posts, while the highest maximum was infectious disease posts. About the number of users adding to favorites, the highest median was environmental health posts, while the highest maximum was vaccine-related event posts.

Table 2 reveals the factors associated with the engagement of followers for posts published by the GZCDC i-Health OWA. The multiple logistic regression model showed that the number of post views was positively correlated to infectious disease posts (AOR: 3.20 95\% CI: 1.16-8.81), original posts (AOR: 10.20, 95\% CI: $1.17-89.28)$, and posts where the title reflected the content (AOR: 2.93, 95\% CI: 1.16-8.81). In addition, the number of reposts was positively associated with nutrition and food-borne disease posts (AOR: 3.57, 95\% CI: 1.24-10.27), chronic disease posts (AOR: 3.26, 95\% CI: 1.03-10.31), headline posts (AOR: $5.01,95 \% \mathrm{CI}=1.36-18.51)$, and posts focused on current events (AOR: $2.11,95 \% \mathrm{CI}=1.02-4.47$ ). The number of adding to favorites had a positive correlation with chronic disease posts (AOR: 4.30, 95\% CI: 1.31-14.13), headline posts (AOR: 6.19, 95\% $\mathrm{CI}=1.62-23.58)$, and posts where the title reflected the content (AOR: 2.43, 95\% CI: 1.12-5.28).

\section{DISCUSSION}

To the best of our knowledge, this is the first study by the CDC to explore the influencing factors on followers' engagement of the OWA of the CDC in Guangzhou, South China. Our study showed that the accumulated number of followers of the GZCDC i-Health OWA increased steadily, which indicated the potential application perspective of the OWA to enhance health information and public health literacy. The volume of post views and shares peaked on the first day of the post, and dropped sharply to the very low level in the following 7 days. The key period for health information and dissemination of health literacy was the first 7 days. Although we did not take the week day and hours of posts into consideration, a prior study showed that most Facebook posts were posted during weekends, and the majority of posts per day fell between midnight and early morning (14). The finding reminded us that posts of the OWA should be posted during this time period to ensure better dissemination and even use certain measures to encourage the public to share the posts, such as a lucky draw with a gift reward. Furthermore, we could use the data of posts to facilitate our infectious disease surveillance. A model study indicated that 1 week-lagged Zika tweets were best correlated with weekly ZIKV cases (27). The application of public account monitoring in the early warning and prediction of infectious diseases should be strengthened in the future.

The median of the top three post views was chronic disease, nutrition and food-borne disease, and infectious disease, which indicated that these topics were more appealing to readers. This finding was almost the same as the prior study, which revealed that articles about infectious disease, food safety and nutrition, vaccination, and health life style were inclined to get more post views (28). It hinted that these kinds of issues should be strengthened in the OWA health information in future. We 

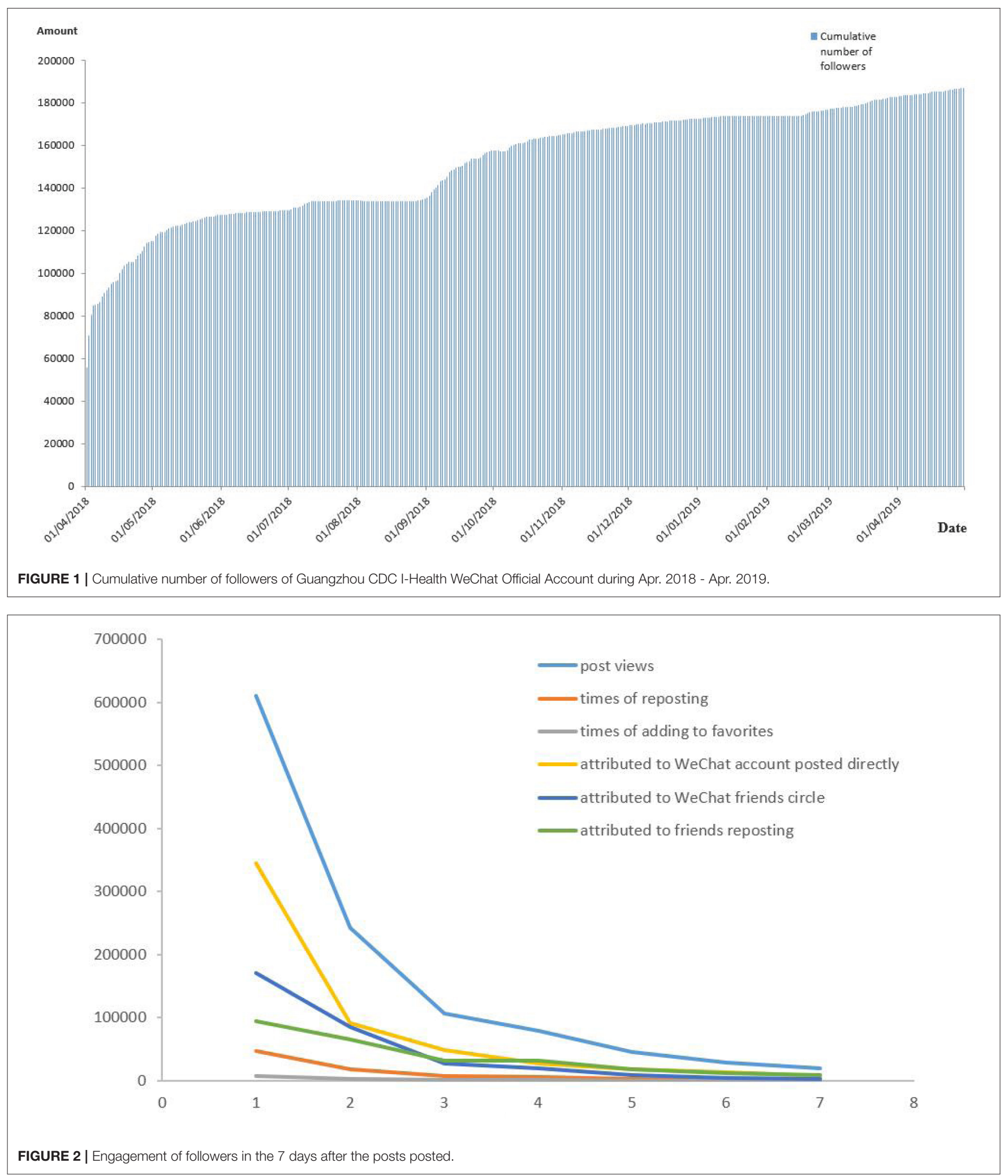

found that most followers of the OWA were women, which was different from other social media. For example, Facebook and Instagram have a relative balance of male and female users, while
Twitter gets more male users. It may be attributed to the fact that women were more interested in gaining health information than men. Women are known to be some of the most prolific social 
TABLE 1 | Engagement of followers for the posts posted by the Guangzhou CDC I-Health official WeChat account.

\begin{tabular}{|c|c|c|c|c|c|c|c|c|c|c|c|c|c|c|c|c|}
\hline \multirow[t]{3}{*}{ Variables } & \multirow[t]{3}{*}{$n(\%)$} & \multicolumn{5}{|c|}{ Post views } & \multicolumn{5}{|c|}{ Share } & \multicolumn{5}{|c|}{ Add to favorites } \\
\hline & & \multicolumn{3}{|c|}{ Percentile } & \multirow[t]{2}{*}{ Min. } & \multirow[t]{2}{*}{ Max. } & \multicolumn{3}{|c|}{ Percentile } & \multirow[t]{2}{*}{ Min. } & \multirow[t]{2}{*}{ Max. } & \multicolumn{3}{|c|}{ Percentile } & \multirow[t]{2}{*}{ Min. } & \multirow[t]{2}{*}{ Max. } \\
\hline & & 25 & 50 & 75 & & & 25 & 50 & 75 & & & 25 & 50 & 75 & & \\
\hline \multicolumn{17}{|l|}{ TYPE OF POSTS } \\
\hline Organizational promotion & $50(23.47)$ & 634.5 & 1203.5 & 2656.5 & 314 & 64,881 & 15.5 & 61.5 & 161.25 & 0 & 7,540 & 0 & 3 & 9.5 & 0 & 176 \\
\hline Infectious disease & $61(29.64)$ & 2,150 & 4,054 & 7,449 & 803 & 98,823 & 83 & 169 & 359 & 11 & 11,400 & 3 & 7 & 27 & 0 & 245 \\
\hline Vaccine-associated event & $21(9.86)$ & 1325.5 & 3,946 & 7596.5 & 452 & 33,852 & 70.5 & 179 & 477 & 6 & 4,782 & 6 & 12 & 56.5 & 0 & 834 \\
\hline Environment health & $16(7.51)$ & 1844.75 & 3,491 & 5279.75 & 891 & 33,722 & 101.75 & 220.5 & 370 & 41 & 1,546 & 10.25 & 21.5 & 36.75 & 2 & 98 \\
\hline Nutrition and food-borne disease & 35 (16.43) & 2892.25 & 4,229 & 6317.25 & 1,356 & 14,537 & 121.25 & 242 & 377.5 & 39 & 897 & 5.25 & 11 & 21.75 & 2 & 54 \\
\hline Chronic disease & $25(11.74)$ & 2,797 & 4,733 & 6,221 & 729 & 11,530 & 121 & 216 & 435.5 & 32 & 826 & 5.5 & 16 & 37 & 0 & 114 \\
\hline Child and adolescent health & $5(2.35)$ & 3001.5 & 3,790 & 8878.5 & 2,672 & 13,666 & 132 & 247 & 706.5 & 71 & 1,049 & 7.5 & 13 & 104.5 & 6 & 195 \\
\hline \multicolumn{17}{|l|}{ CHARACTERISTIC OF POSTS } \\
\hline Original post & $195(91.55)$ & 2,013 & 3,929 & 6,389 & 314 & 98,823 & 79 & 182 & 385 & 0 & 11,400 & 3 & 11 & 26 & 0 & 834 \\
\hline Repost & $18(8.45)$ & 893 & 1119.5 & 1842.75 & 452 & 4,036 & 38.25 & 74 & 96.25 & 3 & 557 & 0.75 & 3.5 & 8.25 & 0 & 51 \\
\hline \multicolumn{17}{|l|}{ EFFECTIVENES } \\
\hline Focused on hot spots & $48(22.54)$ & 2434.75 & 3,929 & 7159.75 & 836 & 64,881 & 126.75 & 220 & 600.5 & 20 & 7,540 & 6 & 11 & 25.25 & 1 & 313 \\
\hline Common event & $165(77.46)$ & 1504.5 & 3,306 & 5957.5 & 314 & 98,823 & 58.5 & 155 & 349 & 0 & 11,400 & 2 & 9 & 23 & 0 & 834 \\
\hline \multicolumn{17}{|l|}{ HEADLINE } \\
\hline Yes & $186(87.32)$ & 2356.25 & $4,040.5$ & 6,560 & 475 & 98,823 & 96.75 & 191 & 397.5 & 3 & 11,400 & 5 & 11 & 27 & 0 & 834 \\
\hline No & 27 (12.68) & 502 & 729 & 912 & 314 & 1,804 & 7 & 18 & 42 & 0 & 557 & 0 & 0 & 4 & 0 & 67 \\
\hline
\end{tabular}

TABLE 2 | Factors associated with engagement of followers on posts by the Guangzhou CDC I-Health official WeChat account.

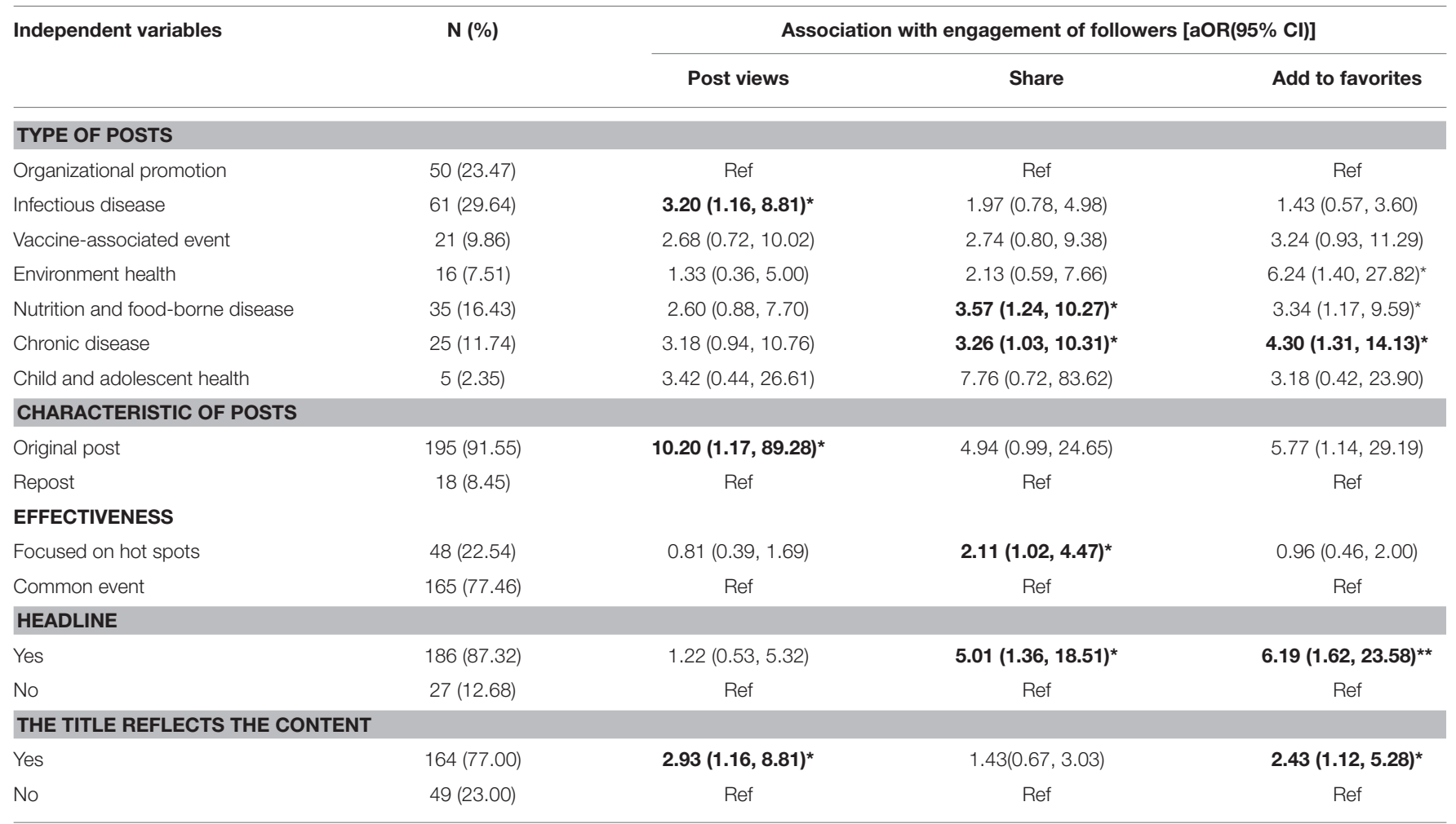

$A O R$, adjusted odds ratio.

${ }^{*} p<0.05,{ }^{* *} p<0.01$.

The bold values meant that they are statistically significant. In other words, the $P$ value is less than $0.05,0.01$ or 0.001 . 
media users (1). This may also contribute to the higher rates of engagement $(29,30)$. Meanwhile, we should find ways to attract more male readers to expand the influence and effectiveness of health information.

Our analysis showed several characteristics of posts that were positively associated with health information dissemination. The headline was positively associated with the amount of shares, and adds to favorites. A recent study during the COVID-19 outbreak in China manifested that the headline played a positive role in a post's popularity (31). It reminded us that we should use the headline to transmit the most important and even emergency health information for better dissemination.

Social media engagement is one of the indicators for the effectiveness of health information (32). A study focused on Facebook revealed strong associations between health education posts and risk communication posts with good engagement rates (14). Different types of posts have different features that affect the engagement of the followers of an OWA. The content of articles was correlated to the users' engagement and was identified as an essential factor to determine whether WeChat users forward or share articles with friends (33). Studies of other social media platforms have also shown that the content of posts appears to have a significant effect on user engagement (22). In our study, people tended to share chronic disease and nutrition and food-borne disease posts, while infectious diseases posts had a more positive association with post views than organizational promotion posts. Original posts contributed to higher post views, which reminds us to use original posts in health information as much as possible. Adding to favorites meant that the article was useful to the readership, which is positively associated with the headline, title-reflected content, and type of posts with chronic disease. Organizational promotion was set as the reference in our research, and some studies pointed out that many health organizations were still focused on "pushing" organizational promotion posts to users, rather than encouraging participation and engagementrelated content on social media $(34,35)$. A previous study showed that organizational promotion posts were correlated to low engagement rates. This phenomenon could be attributed to the fact that such posts focus on organization interest more than public interest (36). The features mentioned above suggested that we should choose an appropriate combination to improve the effectiveness of health information dissemination. Meanwhile, it is important to pay attention to methods of delivering messages. Multimedia applications, such as graphics, video, or pictures, make them more accessible to the public when understanding health information (31). However, the engagement was not the same as actual health-related behavior, and the relation between them is still to be investigated in the future.

In addition, the current results revealed that popular science articles were also popular with users, such as an article entitled "Running hot spots." Our study showed that people liked to share posts with hot spots to their moments and friends, however it did not result in or contribute to higher amounts of post views and adds to favorites. A previous study demonstrated that articles with just hot spots were less likely to obtain high-level reading and liking than those with none (28). In other words, it implied that posts with hot spots should still be combined with high quality content (31), headlines, and other marketing elements (28) to achieve a better propagation effect. We should conduct secondary dissemination of health information with propaganda efforts, such as efforts related to major livelihood projects, making use of the advantages of traditional media with a solid audience base, to gain wider attention (37-39).

Our study has certain limitations. First, it was a crosssectional study, so it could not obtain the causal correlation. Second, our results were from the OWA of the Guangzhou Center for Disease Control and Prevention, so it could not represent that of other provinces or cities, and our sample size was limited, which will lead to a wide confidence interval. Third, it was not possible for us to eliminate the likelihood that some potential confounding factors existed in our research, including demographic factors, communication skills, geographical factors, the status of the epidemic, and preventive measures, moreover the effect of number of figures on health information was not taken into consideration in our study.

\section{CONCLUSIONS}

In the current study, we conducted an analysis of the official GZCDC WeChat account, including its post content, composition of followers, and engagement of followers, to understand reading preferences, habits, and associated factors. We should seize the first 7 days for health information dissemination and try to attract more male readers to expand the influence and effectiveness of health information. Meanwhile, we could take advantage of the headline to transmit the most important health information for better dissemination, especially combined with hot spots content.

Our findings elaborated the current status of the OWA of the GZCDC, and facilitate government departments to formulate better strategies and improve the effectiveness for public health information dissemination. Moreover, our results of the CDC's WeChat study could provide a hint for health information launched in other social media platforms around the world, such as Facebook, Instagram, and Twitter.

\section{DATA AVAILABILITY STATEMENT}

The raw data supporting the conclusions of this article will be made available by the authors, without undue reservation.

\section{AUTHOR CONTRIBUTIONS}

WL: conceptualization and writing-review and editing. XM and JL: data curation, methodology, and writing-original draft. $\mathrm{XM}$ and WL: supervision and validation. JL: visualization. 
All authors contributed to the article and approved the submitted version.

\section{FUNDING}

This study was supported by the Medical Health Technology Project for Guangzhou (20201A010045, 20201A011061, 20201A011062), the Key Project of Medicine Discipline of

\section{REFERENCES}

1. Center. DMPR. The Demographics of Social Media Users 2015 (2021). Available online at: http://www.pewinternet.org/2015/08/19/the-demographics-ofsocial-media-users/

2. Freeman B, Potente S, Rock V, McIver J. Social media campaigns that make a difference: what can public health learn from the corporate sector and other social change marketers? Public Health Res Pract. (2015) 25:e2521517. doi: 10.17061/phrp2521517

3. Edney S, Bogomolova S, Ryan J, Olds T, Sanders I, Maher C. Creating engaging health promotion campaigns on social media: observations and lessons from Fitbit and Garmin. J Med Internet Res. (2018) 20:e10911. doi: 10.2196/10911

4. Li W, Han LQ, Guo YJ, Sun J. Using WeChat official accounts to improve malaria health literacy among Chinese expatriates in Niger: an intervention study. Malar J. (2016) 15:567. doi: 10.1186/s12936-016-1621-y

5. Zhou J, Liu F, Zhou H. Understanding health food messages on Twitter for health literacy promotion. Perspect Public Health. (2018) 138:1739. doi: 10.1177/1757913918760359

6. Freeman B, Kelly B, Baur L, Chapman K, Chapman S, Gill T, et al. Digital junk: food and beverage marketing on Facebook. Am J Public Health. (2014) 104:e56-64. doi: 10.2105/AJPH.2014.302167

7. Platt T, Platt J, Thiel DB, Kardia SL. Facebook advertising across an engagement spectrum: a case example for public health communication. JMIR Public Health Surveill. (2016) 2:e27. doi: 10.2196/publichealth.5623

8. Karim S, He H, Mallah GA, Laghari AA, Larik R. The impact of using Facebook on the academic performance of university students. In: International Conference on Artificial Intelligence and Security. Cham: Springer (2019). p. 405-18. doi: 10.1007/978-3-030-24271-8_37

9. Zhang Z, Ahmed W. A comparison of information sharing behaviours across 379 health conditions on Twitter. Int J Public Health. (2019) 64:43140. doi: 10.1007/s00038-018-1192-5

10. Guidry JPD, Jin Y, Orr CA, Messner M, Meganck S. Ebola on Instagram and Twitter: how health organizations address the health crisis in their social media engagement. Public Relat Rev. (2017) 43:477-86. doi: 10.1016/j.pubrev.2017.04.009

11. Gauthier TP, Spence E. Instagram and clinical infectious diseases. Clin Infect Dis. (2015) 61:135-6. doi: 10.1093/cid/civ248

12. Zhang X, Wen D, Liang J, Lei J. How the public uses social media WeChat to obtain health information in china: a survey study. BMC Med Informat. Decis Making. (2017) 17:66. doi: 10.1186/s12911-017-0470-0

13. Shafiq $M, Y u X$, Laghari AA. WeChat traffic classification using machine learning algorithms and comparative analysis of datasets. Int J Inf Comput Security. (2018) 10:109. doi: 10.1504/IJICS.2018.091467

14. Rahim AIA, Ibrahim MI, Salim FNA, Ariffin MAI. Health information engagement factors in Malaysia: a content analysis of Facebook use by the Ministry of Health in 2016 and 2017. Int J Environ Res Public Health. (2019) 16:591. doi: 10.3390/ijerph16040591

15. Shaikh ZA, Sathio AA, LaghariAA, Memon MA, Hussain I. Study of the role of new technologies in pharmaceutical industry. J Pharm Res Int. (2019) 31:1-11. doi: 10.9734/jpri/2019/v31i630313

16. Facebook Engagement: Ways to Increase It (2020). Available online at: https:// metricool.com/what-is-facebook-engagement/

17. Tu S, Yan X, Jie K, Ying M, Huang C. WeChat: an applicable and flexible social app software for mobile teaching. Biochem Mol Biol Educ. (2018) 46:555-60. doi: 10.1002/bmb.21170
Guangzhou (No. 2021-2023-11), and the Medical Science and Technology Foundation of Guangdong Province (A2021372).

\section{ACKNOWLEDGMENTS}

We would like to thank the colleagues who contributed to the posts published in the official WeChat account of the Guangzhou Center for Disease Control and Prevention.

18. Cao B, Liu C, Durvasula M, Tang W, Pan S, Saffer AJ, et al. Social media engagement and HIV testing among men who have sex with men in China: a Nationwide Cross-Sectional Survey. J Med Internet Res. (2017) 19:e251. doi: 10.2196/jmir.7251

19. Grajales FJ III, Sheps S, Ho K, Novak-Lauscher H, Eysenbach, G. Social media: a review and tutorial of applications in medicine and health care. $J$ Med Internet Res. (2014) 16:e13. doi: 10.2196/jmir.2912

20. Li WHC, Ho KY, Lam KKW, Wang MP, Cheung DYT, Ho LLK, et al. A study protocol for a randomised controlled trial evaluating the use of information communication technology (WhatsApp/WeChat) to deliver brief motivational interviewing (i-BMI) in promoting smoking cessation among smokers with chronic diseases. BMC Public Health. (2019) 19:1083. doi: 10.1186/s12889-019-7417-6

21. Sumner SA, Bowen DA, Bartholow B. Factors associated with increased dissemination of positive mental health messaging on social media. Crisis. (2020) 41:141-5. doi: 10.1027/0227-5910/a000598

22. Card KG, Lachowsky N, Hawkins BW, Jollimore J, Baharuddin F, Hogg RS. Predictors of Facebook user engagement with health-related content for gay, bisexual, and other men who have sex with men: content analysis. JMIR Public Health Surveill. (2018) 4:e38. doi: 10.2196/publichealth.8145

23. Romero-Rodríguez JM, Rodríguez-Jiménez C, Ramos Navas-Parejo M, Marín-Marín JM, Gómez-García G. Use of Instagram by pre-service teacher education: smartphone habits and dependency factors. Int J Environ Res Public Health. (2020) 17:4097. doi: 10.3390/ijerph17114097

24. Shen L, Wang S, Chen W, Fu Q, Evans R, Lan F, et al. Understanding the function constitution and influence factors on communication for the WeChat official account of top tertiary hospitals in china: cross-sectional study. J Med Internet Res. (2019) 21:e13025. doi: 10.2196/13025

25. Wutzke S, Morrice E, Benton M, Wilson A. What will it take to improve prevention of chronic diseases in Australia? A case study of two national approaches. Aust Health Rev. (2017) 41:176-81. doi: 10.1071/AH16002

26. El Tantawi M, Bakhurji E, Al-Ansari A, AlSubaie A, Al Subaie HA, AlAli A. Indicators of adolescents' preference to receive oral health information using social media. Acta Odontol Scand. (2019) 77:2138. doi: 10.1080/00016357.2018.1536803

27. Masri S, Jia J, Li C, Zhou G, Lee MC, Yan G, Wu J. Use of Twitter data to improve Zika virus surveillance in the United States during the 2016 epidemic. BMC Public Health. (2019) 19:761. doi: 10.1186/s12889-019-7103-8

28. Zhang Y, Xia T, Huang L, Yin M, Sun M, Huang J, et al. Factors influencing user engagement of health information disseminated by Chinese provincial centers for disease control and prevention on WeChat: observational study. JMIR mHealth uHealth. (2019) 7:e12245. doi: 10.2196/12245

29. Karimkhani C, Connett J, Boyers L, Quest T, Dellavalle RP. Dermatology on instagram. Dermatol Online J. (2014) 20:13030/qt71g178w9. doi: 10.5070/D3207023129

30. He C, Wu S, Zhao Y, Li Z, Zhang Y, Le J, et al. Social media-promoted weight loss among an occupational population: cohort study using a WeChat mobile phone app-based campaign. J Med Internet Res. (2017) 19:e357. doi: 10.2196/jmir.7861

31. Ma R, Deng Z, Wu M. Effects of health information dissemination on user follows and likes during COVID-19 outbreak in China: data and content analysis. Int J Environ Res Public Health. (2020) 17:5081. doi: 10.3390/ijerph17145081

32. Heldman AB, Schindelar J, Weaver JB. Social media engagement and public health communication: implications for public health organizations 
being truly "Social". Public Health Rev. (2013) 35:13. doi: 10.1007/BF033 91698

33. Ji H. Analysis of influencing factors of WeChat public information transmission. J Jimei Univ. (2016) 19:107-15.

34. Tursunbayeva A, Franco M, Pagliari C. Use of social media for e-Government in the public health sector: a systematic review of published studies. Gov Inf Quart. (2017) 34:270-82. doi: 10.1016/j.giq.2017.04.001

35. Ramanadhan S, Mendez SR, Rao M, Viswanath K. Social media use by community-based organizations conducting health promotion: a content analysis. BMC Public Health. (2013) 13:1129. doi: 10.1186/1471-2458-13-1129

36. Bhattacharya S, Srinivasan P, Polgreen P. Social media engagement analysis of U.S. Federal health agencies on Facebook. BMC Med Informat Decis Making. (2017) 17:49. doi: 10.1186/s12911-017-0447-z

37. Littlejohns LB, Baum F, Lawless A, Freeman T. Disappearing health system building blocks in the health promotion policy context in South Australia (2003-2013). Crit Public Health. (2019) 29:228-40. doi: 10.1080/09581596.2017.1418501

38. Ebrahimpour A, Rajabali F, Yazdanfar F, Azarbad R, Nodeh MR, Siamian $\mathrm{H}$, et al. Social network sites as educational factors. Acta Informatica Medica. (2016) 24:134-8. doi: 10.5455/aim.2016.24.1 34-138
39. Tang CS, Koh YY. Online social networking addiction among college students in Singapore: comorbidity with behavioral addiction and affective disorder. Asian J Psychiatry. (2017) 25:175-8. doi: 10.1016/j.ajp.2016.10.027

Conflict of Interest: The authors declare that the research was conducted in the absence of any commercial or financial relationships that could be construed as a potential conflict of interest.

Publisher's Note: All claims expressed in this article are solely those of the authors and do not necessarily represent those of their affiliated organizations, or those of the publisher, the editors and the reviewers. Any product that may be evaluated in this article, or claim that may be made by its manufacturer, is not guaranteed or endorsed by the publisher.

Copyright (c) $2021 \mathrm{Ma}, \mathrm{Lu}$ and Liu. This is an open-access article distributed under the terms of the Creative Commons Attribution License (CC BY). The use, distribution or reproduction in other forums is permitted, provided the original author(s) and the copyright owner(s) are credited and that the original publication in this journal is cited, in accordance with accepted academic practice. No use, distribution or reproduction is permitted which does not comply with these terms. 